\title{
Chronic myeloid leukemia following repeated diagnostic $X$-ray exposure for the treatment of recurrent spontaneous pneumothorax in a patient with ankylosing spondylitis: A case report and literature review
}

\author{
FANG-HE JU ${ }^{1}$, XU-BO GONG ${ }^{2}$, TING-ZHEN XU ${ }^{1}$, LI-BIN JIANG ${ }^{1}$, HUI-HUA HONG ${ }^{1}$ and ZHEN WANG ${ }^{1}$ \\ ${ }^{1}$ Department of Respiratory Medicine, Zhejiang Provincial Hospital of Traditional Chinese Medicine, \\ Hangzhou, Zhejiang 310006; ${ }^{2}$ Department of Clinical Laboratory, The Second Affiliated Hospital, \\ School of Medicine, Zhejiang University, Hangzhou, Zhejiang 310009, P.R. China
}

Received May 5, 2017; Accepted September 7, 2017

DOI: $10.3892 / \mathrm{ol} .2017 .7156$

\begin{abstract}
Previous studies have indicated that X-ray irradiation may increase the risk of chronic myeloid leukemia (CML), and the incidence of spontaneous pneumothorax in patients with ankylosing spondylitis (AS) is higher than in the general population. Patients with AS usually develop spontaneous pneumothorax several years after the diagnosis of AS. The present study reports the unusual case and complicated clinical history of a 29-year-old man with recurrent pneumothorax and $\mathrm{AS}$, who developed $\mathrm{CML}$ following repeated exposure to low doses of radiation via diagnostic X-rays and chest computed tomography imaging. Pneumothorax was diagnosed prior to AS in this patient; the present case report highlights the importance of recognizing AS as a possible underlying cause of recurrent spontaneous pneumothorax. Patients with AS may be more sensitive to injury via $\mathrm{X}$-ray-derived radiation, and even small diagnostic doses may be associated with CML. Diagnostic X-ray exposure should therefore be limited to reduce the risk of radiation-associated malignancies, including $\mathrm{CML}$, particularly in patients with AS.
\end{abstract}

Correspondence to: Dr Zhen Wang or Dr Fang-He Ju, Department of Respiratory Medicine, Zhejiang Provincial Hospital of Traditional Chinese Medicine, 54 Youdian Road, Hangzhou, Zhejiang 310006, P.R. China

E-mail: wangzhen610@yeah.net

E-mail: jufanghe2001@126.com

Abbreviations: AS, ankylosing spondylitis; CML, chronic myeloid leukemia; CT, computed tomography; WBC, white blood cell

Key words: ankylosing spondylitis, chronic myeloid leukemia, computed tomography, repeated diagnostic X-ray, spontaneous pneumothorax

\section{Introduction}

Ankylosing spondylitis (AS) is a systemic, multifactorial, chronic rheumatic disease that affects the joints of the axial skeleton (1). A thorough physical examination, in conjunction with family history analysis, consideration of the patient's individual medical history, X-ray imaging and serum human leukocyte antigen (HLA)-B27 testing, is required for an accurate diagnosis (2). Previous research has indicated that the incidence of spontaneous pneumothorax in patients with AS is $0.29 \%$, higher than that observed in the general population (1).

Although the underlying etiology of spontaneous pneumothorax remains unknown, it is an uncommon lung disease that disrupts respiration in otherwise healthy men who tend to be tall and thin (3). Spontaneous pneumothorax results from the rupture of a subpleural emphysematous bleb typically located at the apex of the lung, leading to the accumulation of air in the pleural cavity (3). During the course of diagnosis and treatment, X-ray imaging is often utilized in patients with spontaneous pneumothorax (4).

Chronic myeloid leukemia (CML) is characterized by the abnormal growth of mature myeloid cells; it has been associated with translocation involving chromosomes 9 and 22, resulting in the formation of the Philadelphia chromosome $(5,6)$. The cause of this translocation is unknown, although exposure to ionizing radiation is likely to be a risk factor (7). Previous studies have demonstrated a dose-dependent risk of CML in patients with cancer who undergo radiation therapy $(8,9)$ and experience repeated exposure to diagnostic radiography (7).

Our previous report described the case of a patient with AS who was diagnosed with CML following repeated chest radiography and computed tomography (CT) (10); the report focused primarily on a possible association between low-dose $\mathrm{X}$-ray-derived radiation exposure and CML. However, a recent follow-up revealed that this patient experienced mild lower back pain as well as spontaneous pneumothorax during the course of the disease. No prior reports described the simultaneous occurrence of spontaneous pneumothorax and AS followed by CML after diagnostic exposure to low-dose X-rays. 
Table I. Karyotype analysis of the patient.

\begin{tabular}{lccccc}
\hline Date & BCR/ABL1 $^{\mathrm{a}}(\mathrm{p} 210)$ & BCR/ABL1 (copy) & ABL1 (copy) & BCR-ABL1/ABL1 & IS BCR-ABL1/ABL1 \\
\hline Jun 19 2014 & + & $-(\mathrm{P} 230)$ & - & - & - \\
Feb 13 2015 & + & 61.26 & $1.79 \times 10^{4}$ & 0.003 & 0.003 \\
Jun 25 2015 & - & - & $1.63 \times 10^{4}$ & 0 & 0 \\
Oct 19 2015 & - & - & $3.26 \times 10^{4}$ & 0 & 0 \\
\hline
\end{tabular}

${ }^{\mathrm{a} B C R}-\mathrm{ABL} 1$ is an oncogenic protein resulting from fusion of the tyrosine kinase ABL1 with the BCR protein. BCR, breakpoint cluster region; ABL1, Abelson-1; IS, international standard.

Thus, it was surmised that it was important to reanalyze this previous case (10), and to investigate the possible associations between spontaneous pneumothorax and AS, and CML. The present study reviews the unusual presence of CML following repeated diagnostic low-dose X-ray exposure in a patient with recurrent pneumothorax and AS.

\section{Case report}

Our previous report (10) described a case of recurrent spontaneous pneumothorax and the development of CML following repeated exposure to low-dose diagnostic chest radiography and CT. The findings indicated the importance of investigating the potential dangers of low-dose diagnostic radiation. However, that report only included follow-up information up to January 2015 (10). A more recent follow-up revealed that the patient was diagnosed with AS one month later. As the simultaneous occurrence of these two conditions is quite rare, it was considered vital to reanalyze the case.

The patient was a slender man with no history of smoking who had been diagnosed at the Department of Respiratory Medicine, Zhejiang Provincial Hospital of Traditional Chinese Medicine (Hangzhou, China) with spontaneous pneumothorax on May 6, 2013 at the age of 26. Following the insertion and removal of chest tubing, the patient was discharged from hospital. However, the patient then developed recurrent pneumothorax. On May 24, 2013, the patient underwent thoracoscopic surgery for the resection of bullae. Over the 9 months following the initial diagnosis of spontaneous pneumothorax, the patient underwent 10 radiographic examinations and $4 \mathrm{CT}$ scans of the chest, constituting a total effective radiation dose of $11.7 \mathrm{mSv}$. On April 18, 2014 a general health examination revealed that the patient's white blood cell (WBC) count had increased to $13.9 \times 10^{9} / 1$ (normal range, 4-10x10\%/l). On June 6, 2014, the patient's WBC count had increased to $25.4 \times 10^{9} / 1$. Following bone marrow smear testing on June 132014 and a karyotype analysis on June 19, 2014 the patient was diagnosed with CML (10).

On June 28, 2014, the patient began receiving imatinib mesylate at a dose of $400 \mathrm{mg} / \mathrm{day}$ for the treatment of CML. A WBC count revealed that the abnormally high WBC count had begun to decrease on September 4, 2014. An additional karyotype analysis was subsequently performed (Table I) and a second bone marrow smear test on May 5, 2015 revealed no abnormal cells.

The patient continued to receive imatinib mesylate until June 2016. As of January 2015 he had reported no feelings

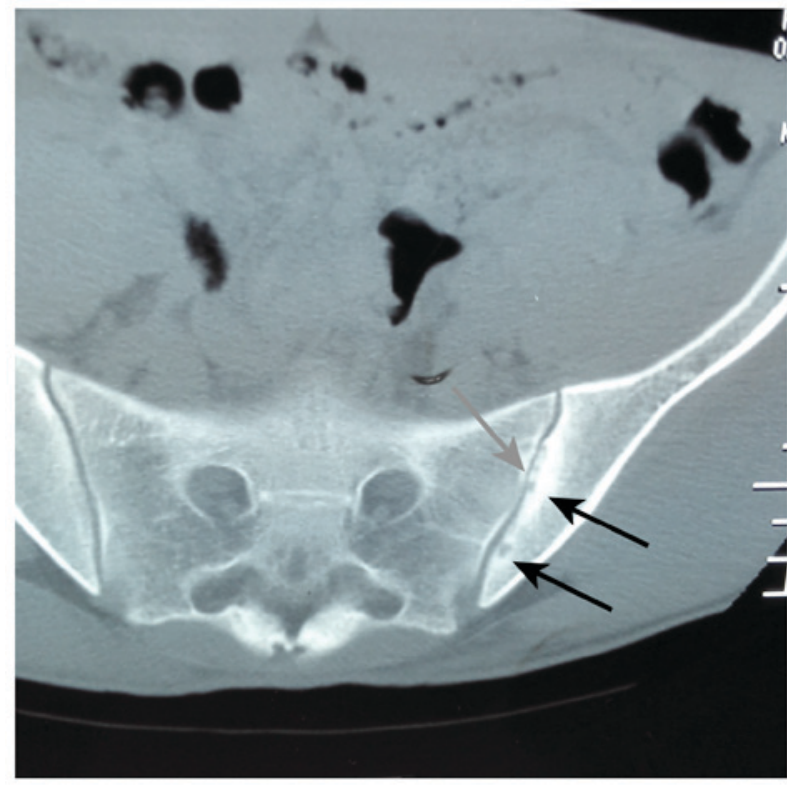

Figure 1. Computed tomography image of the narrowing of the gap in the bilateral sacroiliac joint, and cystic changes in the bilateral iliac bones. The grey arrow indicates narrowing of the sacroiliac joint on the left. Cystic changes and damage were more apparent at the surface of the left sacroiliac joint (black arrows).

of discomfort, as previously described (10). However, the patient did report bilateral sacroiliac joint pain in February 2015. Careful inquiry revealed that the patient had begun to feel intermittent, mild bilateral sacroiliac joint pain as early as January 2014, 6 months prior to the initiation of imatinib treatment. However, the patient had not noted this information because symptoms were mild.

Although the risk of developing CML following repeated exposure to chest radiography and $\mathrm{CT}$ was of concern, a pelvic CT scan was ultimately performed on February 16, 2015 to confirm the diagnosis of AS (Fig. 1). The scan revealed bilateral narrowing of the sacroiliac joints in addition to bilateral cystic changes, which were more apparent at the surface of the left sacroiliac joint.

Joint pain, muscle pain and muscle cramps are known potential side effects of imatinib. In the current case, the location and nature of the pain had not changed after receiving imatinib for 6 months. Moreover, a pelvic CT scan was performed 7 months after the initiation of imatinib treatment. To the best of our knowledge, there have been no prior reports of imatinib 
resulting in AS, thus we hypothesize that AS occurred before the patient was administered imatinib.

The patient tested negative for HLA-B27 and rheumatoid factor, but a diagnosis of AS was reached in accordance with the New York criteria (11). The occurrence of pneumothorax and the subsequent occurrence of CML and AS were documented within the same 2-year period. The Institutional Ethics Committee of the Zhejiang Provincial Hospital of Traditional Chinese Medicine (Hangzhou, China) approved the current study. The subject of this case report provided written informed consent agreeing to publication.

\section{Discussion}

The present study describes the case of a 29-year-old man who presented with recurrent spontaneous pneumothorax, then AS, and who subsequently developed CML following repeated exposure to low-dose diagnostic X-ray radiation. To the best of our knowledge, these combined medical conditions have not been previously reported in the clinical literature.

The patient visited the physician due to pneumothorax; initially, only chest CT scans and chest X-ray data were available. Pelvic CT scanning was not conducted until February 16th, 2015, when it was performed to confirm the diagnosis of AS (Fig. 1). That scan revealed the bilateral narrowing of the sacroiliac joints, in addition to bilateral cystic changes, which were more apparent at the surface of the left sacroiliac joint. The possibility that imatinib caused the occurrence of AS was rejected for the following reasons. Firstly, neither the location nor the nature of the pain changed; secondly, following commencement of imatinib treatment there were no evident changes in the patient's symptoms: Thirdly, the pelvic CT scan was performed just 7 months after the initiation of imatinib treatment; lastly, as no reports of imatinib-induced AS could be located in the literature, we considered this eventuality to be unlikely.

In the majority of cases of unclear etiology, spontaneous pneumothorax is reported in healthy young men and stems from the rupture of apical blebs on the visceral pleura (3). Lee et al (1) reported that the incidence of spontaneous pneumothorax in patients with AS is $0.29 \%(n=1,028)$, higher than that reported for the general population. Although rare, the development of apical pulmonary fibrosis and bullous disease has been recognized as an extra-articular manifestation of AS (12). Studies have also demonstrated that recurrent pneumothorax may be caused by the inadequate sealing of air leaks, and that this can be responsible for the persistence of pneumothorax in patients with connective tissue autoimmune diseases, including AS $(1,13)$.

In prior reports involving AS and concurrent pneumothorax, the pneumothorax was diagnosed a number of years (4-30 years) following the diagnosis of AS $(1,14,15)$. In one previously reported case study, the pneumothorax was diagnosed 16 months after radiation exposure (16). However, the current patient presented with recurrent spontaneous pneumothorax prior to a diagnosis of AS. Notably, previous studies have indicated that the incidence of occult pneumothorax may be underestimated in patients with AS, owing to the vague nature of the symptoms or lack of diagnosis $(1,12)$. Thus, patients with recurrent spontaneous pneumothorax should be thoroughly evaluated for AS.
Specific studies have suggested that rheumatic diseases may develop during the course of CML, although it remains unclear whether the development of rheumatic disease in patients with CML is due to the long-term use of drugs or the underlying disease $(17,18)$. AS is unlikely to be caused by drugs; however, it is imperative to bear in mind that AS, an autoimmune and inflammatory disease, may impair immune function and render patients susceptible to diseases stemming from gene mutations (19). A previous study described a case of CML in a patient with AS following multiple CT imaging sessions (20). Although few reports have documented an association between AS and the development of CML following exposure to low-dose radiation, studies indicate that the risk of hematopoietic cancer is increased in patients with AS (21). The aforementioned findings, together with those of the current case report, indicate that patients with AS may be susceptible to the development of CML following low doses of radiation compared with healthy individuals.

Although blood tests for HLA-B27 are positive in $>90 \%$ of Chinese patients with AS (22), the present patient tested negative for HLA-B27. Numerous studies have also failed to detect an association between HLA-B27 in AS and spontaneous pneumothorax $(1,12,14,15)$. Thus, whether patients with AS who test negative for HLA-B27 are more sensitive to X-ray damage remains to be elucidated. In addition, the potential contributions of sex, age and smoking history to the development of pneumothorax have been reported $(1,12,14,15)$. Therefore, the evidence accumulated to date indicates that further research into the association between spontaneous pneumothorax and sensitivity to radiation in patients with $\mathrm{AS}$ and CML is required.

\section{Acknowledgements}

The authors would like to thank the National Natural Science Foundation of China, Beijing, China for supporting the present study (grant no. 81400107).

\section{References}

1. Lee CC, Lee SH, Chang IJ, Lu TC, Yuan A Chang TA, Tsai KC and Chen WJ: Spontaneous pneumothorax associated with ankylosing spondylitis. Rheumatology (Oxford) 44: 1538-1541, 2005

2. Boos N and Aebi M (eds): Spinal Disorders: Fundamentals of Diagnosis and Treatment. Springer Science+Business Media B.V., Dorhrecht, The Netherlands, 2008.

3. Noppen M: Spontaneous pneumothorax: Epidemiology, pathophysiology and cause. Eur Respir Rev 19: 217-219, 2010.

4. Sahn SA and Heffner JE: Spontaneous pneumothorax. N Engl J Med 342: 868-874, 2000.

5. Konopka JB, Watanabe SM, Singer JW, Collins SJ and Witte ON: Cell lines and clinical isolates derived from Ph1-positive chronic myelogenous leukemia patients express c-abl proteins with a common structural alteration. Proc Natl Acad Sci USA 82: 1810-1814, 1985.

6. Davis RL, Konopka JB and Witte ON: Activation of the c-abl oncogene by viral transduction or chromosomal translocation generates altered c-abl proteins with similar in vitro kinase properties. Mol Cell Biol 5: 204-213, 1985.

7. Preston-Martin S, Thomas DC, Yu MC and Henderson BE: Diagnostic radiography as a risk factor for chronic myeloid and monocytic leukaemia (CML). Br J Cancer 59: 639-644, 1989.

8. Penfold JB and Rhys-Lewis RD: Leukaemia, amyloidosis, and renal vein thrombosis in irradiated ankylosing spondylitis. $\mathrm{Br}$ Med J 2: 1034-1036, 1957.

9. Court-Brown WM and Doll R: Leukaemia and aplastic anaemia in patients irradiated for ankylosing spondylitis. 1957. J Radiol Prot 27: B15-B154, 2007. 
10. Ju FH, Gong XB, Jiang LB, Hong HH, Yang JC, Xu TZ, Chen YU and Wang Z: Chronic myeloid leukaemia following repeated exposure to chest radiography and computed tomography in a patient with pneumothorax: A case report and literature review. Oncol Lett 11: 2398-2402, 2016.

11. van der Linden S, Valkenburg HA and Cats A: Evaluation of diagnostic criteria for ankylosing spondylitis. A proposal for modification of the New York criteria. Arthritis Rheum 27: 361-368, 1984.

12. Rosenow E, Strimlan CV, Muhm JR and Ferguson RH: Pleuropulmonary manifestations of ankylosing spondylitis. Mayo Clin Proc 52: 641-649, 1977.

13. Lawrence RC, Hochberg MC, Kelsey JL, McDuffie FC, Medsger TA Jr, Felts WR and Shulman LE: Estimates of the prevalence of selected arthritic and musculoskeletal diseases in the United States. J Rheumatol 16: 427-441, 1989.

14. Ersoy E, Akgol G and Ozgocmen S: Bilateral spontaneous pneumothorax in a patient with longstanding ankylosing spondylitis. Acta Reumatol Port 39: 353-354, 2014.

15. Wang CT, Tsen JC, Lin HJ and Cheng HH: Bilateral spontaneous pneumothorax in a patient with ankylosing spondylitis. Eur J Emerg Med 14: 123-124, 2007.

16. Bhardwaj H, Bhardwaj B and Youness HA: A case of spontaneous pneumothorax following radiation therapy for non-small cell lung cancer. Lung India 30: 360-362, 2013.
17. Senel S, Kaya E, Aydogdu I, Erkurt MA and Kuku I: Rheumatic diseases and chronic myelogenous leukemia, presentation of four cases and review of the literature. Rheumatol Int 26: 857-861, 2006.

18. Sacchi S, Kantarjian H, O'Brien S, Cohen PR, Pierce S and Talpaz M: Immune-mediated and unusual complications during interferon alfa therapy in chronic myelogenous leukemia. J Clin Oncol 13: 2401-2407, 1995.

19. Linet MS, Slovis TL, Miller DL, Kleinerman R, Lee C, Rajaraman $\mathrm{P}$ and Berrington de Gonzalez A: Cancer risks associated with external radiation from diagnostic imaging procedures. CA Cancer J Clin 62: 75-100, 2012.

20. Ben Abdelghani K, El Menaa M, Ben Abdelghani K, Souabni L, Belhadj S, Chekili S, Laatar A and Zakraoui L: Chronic myeloid leukemia and ankylosing spondylitis. Tunis Med 90: 901, 2012.

21. Thomas E, Brewster DH, Black RJ and Macfarlane GJ: Risk of malignancy among patients with rheumatic conditions. Int J Cancer 88: 497-502, 2000.

22. Ho HH and Chen JY: Ankylosing spondylitis: Chinese perspective, clinical phenotypes, and associated extra-articular systemic features. Curr Rheumatol Rep 15: 344, 2013. 